Revue internationale P.M.E.

Économie et gestion de la petite et moyenne entreprise

\title{
L'innovation technologique dans la très petite entreprise industrielle française : ce que disent les statistiques
}

\section{Jean-Claude Pacitto et Franck Tordjman}

Volume 12, numéro 3, 1999

URI : https://id.erudit.org/iderudit/1008659ar

DOI : https://doi.org/10.7202/1008659ar

Aller au sommaire du numéro

Éditeur(s)

Presses de l’Université du Québec

ISSN

0776-5436 (imprimé)

1918-9699 (numérique)

Découvrir la revue

Citer cette note

Pacitto, J.-C. \& Tordjman, F. (1999). L'innovation technologique dans la très petite entreprise industrielle française : ce que disent les statistiques. Revue internationale P.M.E., 12(3), 59-90. https://doi.org/10.7202/1008659ar
Résumé de l'article

Le particularisme de la très petite entreprise en matière d'innovation est à nuancer. En effet, et par rapport aux indicateurs traditionnels d'innovation, la TPE n'apparaît pas comme une réalité marginale.

Concernant le profil de la TPE innovante, si les statistiques nous renseignent sur bien des aspects reliés aux causes de l'innovation, il n'en demeure pas moins que sur d'autres, et notamment sur les réseaux, les interrogations demeurent et les affirmations doivent être nuancées. 


\section{Notes de recherche L'innovation technologique dans la très petite entreprise industrielle française: ce que disent les statistiques}

Jean-Claude PACITTO

Franck TORDJMAN

Université Paris XII

\section{MOTS CLÉS}

\section{Innovation - TPE - Savoir-faire - Réseau - Recherche-développement}

\section{RÉSUMÉ}

Le particularisme de la très petite entreprise en matière d'innovation est à nuancer. En effet, et par rapport aux indicateurs traditionnels d'innovation, la TPE n'apparaît pas comme une réalité marginale.

Concernant le profil de la TPE innovante, si les statistiques nous renseignent sur bien des aspects reliés aux causes de l'innovation, il n'en demeure pas moins que sur d'autres, et notamment sur les réseaux, les interrogations demeurent et les affirmations doivent être nuancées.

\section{LES AUTEURS}

JeAN-CLAUDE PACITto est maître de conférences et enseigne la stratégie et l'organisation des entreprises à l'Institut universitaire technologique, techniques de commercialisation de l'Université Paris XII. Ses recherches effectuées dans le cadre des activités du Laboratoire IRG Management portent sur la gestion des très petites entreprises. Deux domaines sont de ce point de vue privilégiés: le problème de l'innovation dans les très petites structures et celui de la commercialisation. Adresse: 2, rue de la Fontaine, 89290 Augy, France. Courriel: pacitto@univ-paris12.fr

FRANCK TORDJMAN est professeur agrégé d'économie et gestion à l'IUT de l'Université Paris XII. Il a également été cadre dans des entreprises multinationales de 1983 à 1992 (Procter \& Gamble, Nielsen, Honda). Après avoir connu les très grandes entreprises, ses recherches en cours portent sur la gestion des très petites structures. Adresse : 26, rue du Général-Leclerc, 94220 Charenton, France. Courriel : tordjman@univ-paris12.fr 


\section{ABSTRACT}

The belief in a special relation of tiny enterprises towards innovation has to be qualified. Judging by the traditional innovation indicators, their situation is no exception.

As regards the defining characteristics of the tiny enterprise, statistics provide reliable information on many aspects of the causes of innovation. On other aspects though, particularly networks, some questions remain unanswered, and a few statements need qualifying.

\section{RESUMEN}

La particularidad de la muy pequena empresa in materia de innovacion tecnologica es matizada. En efecto con relacion a los indicatores tradicionales, la MPE no aparece como una realidad marginal. Con respecto a las caractericas de la MPE innovadora, si bien las estatisticas nos informan sobre aspectos relativos a las causas de la innovacion, es claro que sobre otras, y especialmente las redes, quedan preguntas a hacerse y las afirmaciones deben ser relativizadas.

\section{ZUSAMMENFASSUNG}

Bei Innovationen ist die besondere Stellung der Kleinstunternehmungen zu nuancieren. In der Tat ist die Kleinstunternehmung basierend auf den traditionellen Innovationsindikatoren alles andere als eine Randerscheinung.

Auch wenn uns die Statistiken über viele entscheidende Innovationsursachen informieren, spielen die Kleinstunternehmen keine spezielle Rolle. Ihr Profil unterscheidet sich nicht von anderen. Verschiedene Fragen bleiben offen, somit muss die Befürwortung der Sonderrolle der Kleinstunternehmung nuanciert betrachtet werden.

Il est aujourd'hui admis que les innovations de produits ou de procédés se situent au cœur des gains de productivité et influent sur la compétitivité et la croissance des économies industrielles (Guellec, 1993). Le passage d'une économie caractérisée par un schéma fordiste à une économie de variété n'a pas eu comme seule conséquence de raccourcir le cycle de vie des produits mais aussi de transformer en impérative nécessité l'intégration et le développement de technologies toujours en évolution. Source de compétitivité (Dussauge et Ramanantsoa, 1987), la technologie devient aussi facteur de complexité et se trouve posé ici le problème de l'appropriation technologique au cœur des phénomènes de diffusion de l'innovation. Depuis Schumpeter, les économistes n'ont cessé de s'interroger sur la nature de l'innovation, sa causalité et ses effets. Le consensus semble, aujourd'hui, s'être établi sur un refus, celui du modèle linéaire (Kline et Rosenberg, 1986). Toutefois, l'analyse qui tend à apprécier la transformation des inputs en outputs d'innovation, a été, pour une large part, conservée. En ce sens, l'utilisation d'indicateurs s'avère souvent nécessaire, tant pour appréhender l'effort d'innovation que pour évaluer 
les résultats obtenus. Du côté des inputs, les principaux indicateurs retenus pour apprécier l'importance de l'effort en matière de recherche industrielle sont ceux mis au point et « codifiés » par l'OCDE à partir du manuel de Frascati. Ils concernent notamment la mesure de la recherche-développement qui obéit à des définitions strictes. La recherche-développement comprend la recherche fondamentale, qui a pour objet d'acquérir des connaissances sur les fondements des phénomènes et des faits observables sans envisager une utilisation ou une application particulière. La recherche appliquée consiste également en des travaux originaux en vue d'acquérir des connaissances nouvelles mais est dirigée vers un but ou un objectif pratique déterminé. Le développement expérimental consiste en des travaux systématiques basés sur des connaissances existantes en vue de lancer la fabrication de nouveaux produits, d'établir de nouveaux procédés ou d'améliorer considérablement ceux qui existent déjà. Côté outputs, outre le nombre d'innovations, le principal indicateur retenu est le nombre de brevets déposés par les firmes, que l'on peut réagréger par secteur industriel ou pour l'ensemble d'une économie. La pertinence d'utiliser de tels indicateurs est encore en débat (Archibugi, 1988, Smith, 1989). De surcroît, l'application de ces indicateurs aux PME et, dans notre cas, aux TPE s'avère problèmatique ${ }^{1}$. Elles sont en général peu concernées par les mécanismes de propriété industrielle. De la même manière, apprécier leur effort d'innovation à l'aide d'un indicateur de R-D ne revient-il pas à sous-estimer largement leur capacité innovante et à réduire le processus d'innovation à une simple linéarité par ailleurs rejetée ? Enfin, comme le souligne Smith (1989), il est souvent erroné de transformer un raisonnement inductif en raisonnement causal et ce qui apparaît à première vue comme un enchaînement logique est bien souvent le résultat d'interactions complexes, concernant plusieurs variables. En tout état de cause, il n'en demeure pas moins que l'absence quasi totale de données sur la très petite entreprise ne nous permet pas de tester des outils nouveaux et ce qu'il importe d'évaluer, c'est justement le degré de pertinence de ceux existants. Ce n'est qu'après cette première phase que l'on pourra réfléchir à l'élaboration de nouveaux indicateurs et plus généralement d'une nouvelle problématique. Ce sont ces thèmes que nous nous proposons de développer ici en basant notre réflexion sur les résultats de l'enquête TIME (Technological Innovation in Micro Enterprises).

Nous tenterons alors, et au vu des résultats, de montrer ce qui distingue ou ce qui ne distingue pas la très petite entreprise des autres, lorsqu'on s'intéresse à l'innovation technologique et, en particulier, aux sources de l'innovation.

1. Tenant compte du «particularisme » français en matière de très petite entreprise, l'enquête a porté sur les entreprises artisanales de production, entreprises de moins de 10 salariés inscrites au répertoire des métiers. 


\section{Méthodologie de l'enquête et collecte des données}

Les résultats présentés dans l'article sont issus de l'enquête TIME finalisée en 1994 et axée sur le problème de l'innovation technologique.

\subsection{La population concernée}

Les données ont été recueillies dans cinq régions (Centre, Franche-Comté, Île-deFrance, Midi-Pyrénées, Nord-Pas-de-Calais) auprès de 1016 entreprises. Cet échantillon est représentatif, sur la France entière, d'une population d'environ 21000 entreprises définie selon les deux critères suivants :

- présence d'au moins cinq ans dans le répertoire des métiers à un niveau significatif de taille (en chiffre d'affaires).

- appartenance à un secteur de fabrication - notion qui a été étendue aux activités de maintenance au contenu technologique manifeste.

On est ainsi en présence d'entreprises directement concernées par l'innovation technique, comme producteurs ou comme utilisateurs, et qui ont eu le temps d'acquérir une connaissance approfondie de leurs concurrents et de leur marché (même si l'on verra ce que recouvre cette réalité pour la TPE). A contrario, l'enquête n'est pas représentative des problèmes rencontrés par les créateurs d'entreprises, notamment par l'essaimage autour des centres de recherche, ni par les entreprises à courte durée de vie. Une entreprise peut « mourir » statistiquement sans pour autant mourir économiquement ; cette mobilité exclut malheureusement de notre champ un certain nombre d'entreprises dont rien ne permet $a$ priori de dire qu'elles sont plus ou moins innovantes que les autres. D'une manière générale, l'enquête ne vise pas à sélectionner les « vedettes » de l'innovation, mais à donner une image fiable de l'ensemble des secteurs des métiers de production. Pour fixer les idées, les entreprises du champ emploient en moyenne six salariés, possèdent des équipements de production (hors transport) d'une valeur vénale de $850000 \mathrm{~F}$ et réalisent un chiffre d'affaires moyen de cinq millions. Ces moyennes masquent une forte variabilité, due pour une large part à la diversité des activités, mais pas uniquement : un même volume d'affaires peut être réalisé, selon les cas, avec plus ou moins de machines.

\subsection{Structuration des données : variables d'analyse et variables de classification}

Après traitement, la volumineuse masse de données recueillies (1 016 questionnaires validés, soit près d'un millier d'heures d'entretien en entreprise) a été résumée en un petit nombre de variables et d'indicateurs qui concernent la partie utile de l'information. 


\section{Huit indicateurs synthétiques sur l'innovation}

Les performances de l'entreprise en matière de technologie et d'innovation sont décrites par un indicateur unique qui est le «produit central » de l'enquête. Il peut se présenter soit sous forme d'une note variant de zéro à 20 , ce qui permet de calculer des moyennes, soit en cinq classes, ce qui permet un décompte des entreprises, depuis le groupe des « totalement non innovantes » jusqu' à celui des « champions». Cet indicateur tient compte aussi bien des innovations de produits que de celles visant les procédés, des innovations induites par l'emploi de nouvelles machines ou de nouveaux matériaux, des réalisations comme les innovations en cours ou en projet.

Deux autres indicateurs de performance, plus simples, sont également utilisés : la proportion dans le chiffre d'affaires des produits innovants et celle des produits destinés à l'exportation (directement ou indirectement). Cinq autres indicateurs synthétiques ont été établis, tous sous la forme de notes variant de zéro à 20 et de classes d'entreprises. Trois concernent les « intrants » du processus d'innovation (la recherche-développement, l'acquisition d'informations scientifiques et techniques, l'informatique installée dans l'entreprise). Les deux derniers décrivent l'attitude de l'entreprise à l'égard des enjeux technologiques. L'un est construit sur des données objectives, essentiellement les relations entre l'entreprise et ses partenaires, qu'ils soient publics, privés ou institutionnels (tableau 3). Le second est une synthèse des opinions émises par l'artisan sur sa capacité à innover, ses intentions d'investir et le dynamisme de son secteur. Ces grandeurs sont à la fois des intrants et des extrants de l'innovation.

\section{Être et se sentir artisan: neuf variables d'opinion}

À ces huit indicateurs qui répondent à l'objet central de l'enquête s'ajoute un groupe de neuf questions d'opinion qui portent sur les valeurs de référence de l'entreprise artisanale en général (c'est-à-dire indépendamment des problèmes de technologie). Les réponses à ces questions, qui toutes avaient été spontanément formulées au cours de la préparation de l'enquête, montrent des convergences très fortes qui, fait remarquable, n'ont aucune relation avec les performances à l'innovation des entreprises.

\section{Structure de l'activité et des marchés de l'entreprise}

L'innovation n'est pas la nouveauté, mais il existe peu de moyens objectifs permettant d'établir cette distinction. L'enquête ne comprenait pas de questions sur le cycle de vie des produits, mais abordait deux notions importantes pour caractériser l'artisanat de production: 
- la distinction entre produits vendus sur catalogue et produits fabriqués sur devis, permettant également d'isoler les activités de négoce pur, de maintenance ou d'après-vente, ainsi que les études et prototypes. On comprendra que dans chacun des deux cas les notions d'innovation et de nouveauté n'ont pas le même sens ;

- la structure de clientèle de l'entreprise où l'on distingue les activités à fort contenu technologique (spatial, nucléaire, défense).

Deux autres questions peuvent être rattachées à ce groupe : la répartition du capital en trois catégories d'âge (générations technologiques) et selon leur pays de construction.

\section{L'activité, critère de lecture quasi unique}

Ensemble, ces trois groupes constituent les variables d'analyse que l'on va décrire selon les modalités de «variables de classification » que sont les principaux descripteurs de la population étudiée : activité, âge de l'artisan, région, niveau de formation. L'enquête ne permet pas, compte tenu des effectifs interrogés et du déroulement de la collecte, la production de résultats régionaux significatifs. En fait, c'est le secteur d'activité, ici défini en huit postes, de loin le plus discriminant des critères de classification disponibles dans l'enquête, qui sera toujours utilisé seul. Le niveau de formation, à condition d'être ramassé en trois postes, apporte également des indications intéressantes, ainsi que le volume des investissements productifs utilisés dans l'entreprise.

\subsection{Définition des huit regroupements d'activité (total 21000 entreprises)}

La nomenclature d'activités a été définie entre les deux vagues de la collecte de l'enquête, après une analyse des résultats sur 500 entreprises. Nous souhaitions construire des regroupements homogènes d'au moins 100 entreprises pour assurer assez de robustesse aux résultats ; cela n'a pas été possible pour trois activités, qu'il n'aurait pas été pertinent de fusionner, l'effectif le plus faible étant de 76.

1) La mécanique (4 000 entreprises), secteur homogène où l'on travaille le plus souvent sur devis, en utilisant beaucoup d'équipements (valeur moyenne : 1,6 million de francs) et en innovant assez souvent ( $20 \%$ d'entreprises innovantes).

2) Les industries du bois (plus de 2000 entreprises), depuis les scieries jusqu'aux activités de transformation finale, en moyenne assez fortement équipées (1 million de francs) et assez innovantes (17\% d'entreprises innovantes). 
3) Les autres productions à usage professionnel (plus de 4000 entreprises), où ont été regroupées toutes les fabrications de biens d'équipement, en dehors de la mécanique et du travail du bois. On y trouve une grande variété d'activités, entre autres l'électronique, mais sans qu'aucun domaine n'apparaisse dominant. Le niveau d'équipement moyen est important (1,05 million de francs) et c'est le secteur globalement le plus innovant, quel que soit l'indicateur choisi (33\% d'entreprises innovantes). C'est également là que l'on trouve la plus forte proportion de diplômés de l'enseignement supérieur, qu'il s'agisse de l'artisan ou de l'un de ses collaborateurs (30\% des entreprises, contre une moyenne générale de $14 \%$ ).

4) La filière textile-habillement-cuir (1 500 entreprises), où l'on trouve des entreprises très fortement capitalistiques à côté d'autres qui ne le sont pas du tout ; néanmoins, selon d'autres critères, le secteur est trop homogène. L'innovation y est très peu présente, mais la nouveauté et l'exportation sont fréquentes.

5) Les biens de consommation, c'est-à-dire les autres productions destinées aux particuliers (3000 entreprises dont 1200 prothésistes), bien qu'on y trouve $19 \%$ d'entreprises innovantes, le niveau moyen de performance à l'innovation est médiocre. Les équipements sont beaucoup plus légers (0,35 million de francs).

Et trois groupes d'entreprises se consacrant, pour une part importante, à des activités de maintenance, souvent d'ailleurs accompagnées de négoce pur et simple : la machine agricole (moins de 2000 entreprises), la réparation électrique (3000 entreprises) et le génie climatique (1 500 entreprises). La fabrication sur devis y est majoritaire ; ces entreprises, conformément en cela aux règles adoptées pour l'échantillonnage, ont surtout une clientèle d'entreprises ou de collectivités. Le niveau d'équipement moyen y est réduit ( 0,2 million de francs), et si les performances à l'innovation sont largement inférieures à celles des autres secteurs, il faut se garder de comparaisons hâtives, car ces entreprises ont avant tout un rôle de diffusion des technologies. L'ordre de classement des activités dans les tableaux de résultats, toujours le même, suit en gros la séquence des codes correspondants de la nomenclature APE: les biens d'équipement d'abord, puis les biens de consommation, enfin, les services.

\subsection{Lecture des tableaux}

Comme dans le cas des tableaux concernant les impulsions et les sources de l'innovation (tableaux 1, 2 et 4), elles peuvent porter sur une question présentée dans le questionnaire sous forme ordinale et être évaluées selon une logique logarithmique, le résultat moyen pouvant théoriquement varier de 1 à 4 : 


$\begin{array}{ll}\text { Aucune influence } & \text { valeur : } 0 \\ \text { Influence faible } & \text { valeur : 1 } \\ \text { Influence moyenne } & \text { valeur : 2 } \\ \text { Influence forte } & \text { valeur : 4 }\end{array}$

Pour ce qui concerne le tableau 1 , le résultat a été multiplié par 1000 de façon à garder quatre décimales. Pour ce même tableau, nous avons effectué un raccordement de deux enquêtes, ce qui est toujours hasardeux, même si, comme c'est le cas, les questions sont codées de manière identique.

L'enquête industrie s'est faite par voie postale, celle sur la TPE, par visites ; dans le premier cas, les entreprises totalement non innovantes ont des intensités de sources identiquement nulles, alors que dans la seconde enquête elles ont pu répondre si elles le désiraient. Ceci conduit à une discontinuité forte sur la colonne somme et sur la colonne Proto-R-D dont la prédominance est dopée par les déclarations des entreprises les moins actives.

\subsection{La répartition des entreprises les plus innovantes et des entreprises les moins innovantes (tableaux 4, 9 et 10)}

Afin de mieux faire ressortir les différences entre entreprises, et ce, sur un certain nombre de thèmes habituellement traités dans la littérature, il nous a paru souhaitable d'effectuer un tri supplémentaire, dans chaque activité, entre, d'une part, les entreprises innovantes et, d'autre part, les non-innovantes. Il nous est apparu en effet que c'était une méthode qui permettait, au-delà des comparaisons sectorielles, d'appréhender les différences de comportements ou, selon la terminologie habituelle, de trajectoires. Pour ce faire et en fonction de la formule de calcul de l'indicateur synthétique d'innovation, nous avons classé les entreprises qui ont un score s'échelonnant de un à neuf dans les non-innovantes et celles de 10 à 20 , dans les plus innovantes. La première catégorie (près de 700 entreprises) regroupe des entreprises pour qui l'innovation ne constitue pas une priorité et qui ne sont, à aucun degré, engagées dans un processus d'innovation. La seconde catégorie (300 entreprises), au contraire, regroupe des entreprises qui, au minimum, ont réalisé une innovation de procédé et qui se sentent concernées par le problème.

\subsection{Détail de la formule de calcul de l'indicateur d'ouverture sur l'extérieur (tableau 3)}

Recherche-développement externe : 0 à 4 points avec une autre entreprise de production : 4 points avec une autre catégorie de prestataire : 3 points 
Partenariat technologique interentreprises : 0 à 3 points en cours au moment de l'enquête : 3 points ayant existé mais terminé : 2 points

Relations avec l'ANVAR : 0 à 3 points

Relations avec une institution hors ANVAR : 0 à 2 points coopération technique : 2 points recherche d'informations : 1,5 point

Relations sur le financement de l'exportation : 1 point

Relations sur le financement des impayés : 1 point

Existence d'une plaquette : 2 points

Embauche facile de personnel qualifié : 1 point

$\mathrm{Au}$ moins quatre organismes cités hors ANVAR : 1 point

Pénalités possibles : jusqu'à 3 points résultats décevants avec l'ANVAR : 1 point innovation arrêtée pour partenariat déficient : 1 point difficulté d'accès à l'information ressentie comme principal frein psychologique à l'innovation : 1 point

L'application des pénalités ne peut faire descendre la note en dessous de zéro.

\section{Les principaux résultats qui émergent}

\subsection{Les sources de l'innovation}

«D'où les petites entreprises tirent-elles les ressources leur permettant d'innover?» Cette interrogation légitime de deux auteurs (Audretsch et Vivarelli, 1994) pose le problème et souligne le paradoxe suivant : comment, et par rapport aux ressources possédées (faibles), les petites entreprises parviennent-elles à innover ? Car, et c'est un des principaux résultats de l'enquête, les très petites entreprises innovent, et l'activité d'innovation est loin d'être résiduelle. Pour expliquer ce paradoxe, on s'est tourné ces dernières années vers la théorie des réseaux en partant d'un raisonnement simple : les petites entreprises compensent leurs ressources internes insuffisantes par des contacts avec l'extérieur, contacts qui sont, à moyen et long terme, générateurs de nouvelles ressources. De fait, et sans toujours s'en rendre compte, on a quelque peu oublié de se poser et de poser les questions élémentaires. À cela, pensons-nous, deux raisons. La première est de toute évidence le fait que, ces dernières années, la PME a été un moyen utile pour bâtir nombre de paradigmes alternatifs tant en économie qu'en sciences de gestion. La seconde, qui est, n'en doutons pas, la conséquence de la première, est le manque évident, lorsqu'on traite de la petite entreprise et encore plus de la très petite, de données empiriques. En 
effet et en matière d'innovation, seules celles-ci peuvent permettre de conclure à une spécificité de la très petite entreprise. C'est ce travail que nous allons ici tenter en nous servant des données de l'enquête innovation dans la très petite entreprise industrielle qui comble les lacunes, en cette matière, de l'enquête annuelle d'entreprise. Globalement, et au regard des sources de l'innovation, les petites et moyennes entreprises ne diffèrent pas fondamentalement des grandes entreprises ; c'est ce qu'a mis en évidence l'enquête italienne de 1985. Archibugi, Cesaratto et Sirilli (1991) concluent alors : «Dans le cas des sources externes, il y a une relation évidente entre la taille de l'entreprise et l'importance accordée à chaque source. L'acquisition de brevets et de savoir-faire joue un rôle plus significatif dans les grandes entreprises tandis que les biens intermédiaires apparaissent comme le véhicule privilégié des plus petites. » Les résultats des enquêtes françaises (EAE TIME) ne contredisent pas ces conclusions, mais à la différence de l'enquête italienne, les intrants technologiques n'ont pas été intégrés dans les sources externes, ce qui rend plus malaisées les comparaisons.

\section{TABLEAU 1}

Sources de l'innovation

\begin{tabular}{lrrrrrrr}
\hline & $\begin{array}{c}\text { R-D } \\
\text { interne }\end{array}$ & $\begin{array}{c}\text { Proto- } \\
\text { R-D }\end{array}$ & $\begin{array}{c}\text { R-D } \\
\text { externe }\end{array}$ & & Licences Machines & Matériau & Somme \\
\hline Moins de 10 & 750 & 2020 & 400 & 110 & 930 & 990 & 5200 \\
20 à 49 & 624 & 997 & 380 & 186 & 1016 & 727 & 3930 \\
50 à 99 & 970 & 1300 & 537 & 270 & 1189 & 839 & 5105 \\
100 à 199 & 1302 & 1566 & 660 & 297 & 1356 & 965 & 6146 \\
200 à 499 & 1713 & 1715 & 851 & 405 & 1460 & 1022 & 7166 \\
500 à 999 & 2300 & 2043 & 1208 & 490 & 1772 & 1318 & 9131 \\
1000 à 1999 & 2702 & 2353 & 1326 & 585 & 1827 & 1439 & 10232 \\
2000 et plus & 2961 & 2451 & 1614 & 690 & 2157 & 1742 & 11615 \\
\hline
\end{tabular}

Source : IRG, 1995.

La distinction entre grandes, petites et moyennes entreprises réside surtout dans la diversification des sources utilisées. Ainsi, plus une entreprise est grande, plus elle diversifie ses sources d'innovation en achetant de la technologie à l'extérieur et, notamment, au groupe auquel elle est liée. Pour innover, les grandes entreprises font massivement appel à leur recherche permanente et à la R-D soustraitée. L'achat de technologie incorporée, bien que quasi systématique pour les grandes industries, joue un rôle moins important que pour les plus petites entreprises. Il est aussi intéressant de constater que l'intensité de chaque source diminue avec la taille de l'entreprise. Mais le rythme de cette diminution peut être très différent d'une source à l'autre. À l'inverse, la R-D a un effet très lié à la taille de l'entreprise : au-dessus de 500 salariés, la R-D est dominante; à 500 salariés, elle s'affaisse au niveau de Proto-R-D ; à 200 salariés, au niveau des nouvelles machines et à 50 
salariés, au-dessous des nouveaux matériaux. Si l'on intègre, dans cette comparaison, les très petites entreprises, on constate qu'une inversion s'est produite sur les intrants. Alors que la PMI privilégie les matériels par rapport aux matériaux et composants, la très petite entreprise se caractérise, elle, par une préférence pour ce dernier. Il convient de noter, pour ce qui concerne la TPE, que l'intrant « nouveaux matériaux » constitue la seconde source de l'innovation après le savoir-faire interne (Proto-R-D). Si l'on en suit la catégorisation élaborée par Rosenberg (1982), on retrouve évidemment l'apprentissage par l'usage difficilement dissociable de l'apprentissage par l'interaction tant les contacts avec les fournisseurs et l'utilisation effective de leurs produits sont liés. Il ne fait aucun doute qu'il s'agit là d'une spécificité de la TPE dont il faut tenir compte surtout lorsqu'on s'interroge sur les politiques publiques à mettre en œuvre pour aider la TPE. Pour autant, cette spécificité n'est pas rupture, car non exclusive d'autres sources de l'innovation, et bien sûr de la R-D. À cet égard, l'examen attentif des sources de l'innovation (tableau 2) dans la TPE révèle des résultats intéressants.

TABleau 2

Sources de l'innovation (intensités moyennes)

\begin{tabular}{|c|c|c|c|c|c|c|c|c|c|}
\hline Activité & $\begin{array}{l}\text { S1: } \\
\text { R-D } \\
\text { interne }\end{array}$ & $\begin{array}{l}\text { S2: } \\
\text { Savoir- } \\
\text { faire } \\
\text { interne }\end{array}$ & $\begin{array}{l}\text { S3: } \\
\text { R-D } \\
\text { externe }\end{array}$ & $\begin{array}{l}\text { S4: } \\
\text { Brevets } \\
\text { et } \\
\text { licences }\end{array}$ & $\begin{array}{l}\text { S5: } \\
\text { Infor- } \\
\text { mation } \\
\text { organisée }\end{array}$ & $\begin{array}{l}\text { S6: } \\
\text { Infor- } \\
\text { mation } \\
\text { informelle }\end{array}$ & $\begin{array}{l}\text { S7: } \\
\text { Nouvelles } \\
\text { machines } \\
\text { e }\end{array}$ & $\begin{array}{l}\text { S8: } \\
\text { Nouvelles } \\
\text { compé- } \\
\text { tences }\end{array}$ & $\begin{array}{l}\text { S9: } \\
\text { Nouveaux } \\
\text { matériaux }\end{array}$ \\
\hline $\begin{array}{l}\text { Mécanique } \\
\text { générale }\end{array}$ & 0,80 & 2,37 & 0,39 & 0,10 & 0,63 & 1,02 & 1,49 & 0,56 & 0,71 \\
\hline $\begin{array}{l}\text { Matériel } \\
\text { agricole } \\
\text { Biens }\end{array}$ & 0,60 & 1,54 & 0,32 & 0,20 & 1,46 & 0,81 & 0,62 & 0,46 & 0,57 \\
\hline $\begin{array}{l}\text { professionnels } \\
\text { Industries }\end{array}$ & 1,35 & 2,52 & 0,51 & 0,19 & 0,74 & 1,14 & 1,14 & 0,86 & 1,36 \\
\hline $\begin{array}{l}\text { du bois } \\
\text { Textile, }\end{array}$ & 0,62 & 2,13 & 0,32 & 0,10 & 0,48 & 0,75 & 1,06 & 0,68 & 0,97 \\
\hline & 0,39 & 1,55 & 0,35 & 0,05 & 0,33 & 0,47 & 0,85 & 0,37 & 0,76 \\
\hline $\begin{array}{l}\text { consommation } \\
\text { Installation }\end{array}$ & 0,83 & 1,93 & 0,49 & 0,15 & 0,78 & 0,93 & 0,79 & 0,52 & 1,10 \\
\hline $\begin{array}{l}\text { électrique } \\
\text { Génie }\end{array}$ & 0,29 & 1,50 & 0,29 & 0,01 & 0,74 & 0,75 & 0,40 & 0,41 & 1,00 \\
\hline $\begin{array}{l}\text { climatique } \\
\text { Ensemble }\end{array}$ & 0,30 & 1,68 & 0,38 & 0,04 & 0,78 & 0,69 & 0,43 & 0,37 & 1,11 \\
\hline du champ & 0,75 & 2,02 & 0,40 & 0,11 & 0,65 & 0,89 & 0,93 & 0,57 & 0,99 \\
\hline
\end{tabular}

Source : Enquête TIME.

En premier lieu, il convient de constater que l'activité de recherche-développement constitue, même pour les très petites structures, une des premières sources de l'innovation. Ce résultat représente un premier démenti des perspectives schumpéteriennes. En second lieu, si, à l'instar des grandes entreprises, les sources 
internes devancent les sources externes, en revanche, il est important de souligner que la TPE se distingue de l'ensemble des entreprises - y compris des PME - en ceci que, toutes tailles d'entreprises confondues, la seule R-D interne constitue la troisième source de l'innovation, et la quatrième, pour les PME dont l'effectif est compris entre 20 et 49 salariés; elle constitue la première source dans les plus grandes entreprises et n'est que la cinquième source dans les TPE.

Toutes tailles d'entreprises confondues, la seule R-D strictement externe (hors R-D acquise dans le groupe) constitue la sixième source de l'innovation et la cinquième dans les seules PME dont l'effectif est compris entre 20 et 49 salariés. Elle n'est que l'avant-dernière source de l'innovation dans la très petite entreprise.

À la lecture des résultats, il convient de tempérer l'affirmation de principe visant à ériger la seule R-D en source maîtresse de l'innovation technologique dans la TPE. On constate en effet que les intensités moyennes de chacune des sources de l'innovation sont réparties sinon de manière homogène, tout au moins sans que l'une des sources identifiées ne se détache nettement des autres intrants technologiques, sauf dans le cas particulier du savoir-faire interne. Ce phénomène confirme implicitement que le processus d'innovation n'obéit pas strictement à un modèle linéaire mais plutôt, comme l'ont mis en évidence Kline et Rosenberg (1986), à un mécanisme d'interactions dans lequel l'ensemble des intrants sont enchevêtrés, liés par une série de boucles avec des phénomènes de capitalisation partielle des stocks de connaissances à diverses étapes du processus de production. Toutefois, et comme l'avait suggéré Kleinknecht et Reijnen (1991), la petite entreprise n'ignore pas la recherche-développement, pour autant que le questionnement visant celle-ci soit adapté, ce qui ne signifie pas reconstruit. Archibugi, Evangelista et Simonetti (1995) ont, par ailleurs, montré que la recherche-développement ne constituait qu'un des éléments des coûts que l'entreprise assume pour innover; cette notion de coût d'innovation permet de mieux appréhender la nature des inputs et d'élargir la classification traditionnelle.

La difficulté fondamentale pour la TPE, du fait de sa faible différenciation, provient de son mode d'apprentissage. Comment le dirigeant parvient-il à intégrer ces différents savoirs ? Par quels mécanismes sont-ils fusionnés ? Car c'est ici une autre difficulté : la TPE, peut-être plus que les autres, ne distingue pas les divers modes d'apprentissage. Chacun se trouve mêlé à l'autre sans que l'on puisse les dissocier; en ce sens, il nous est impossible de distinguer l'apprentissage par l'action de l'apprentissage par la pratique. Difficulté aggravée par le caractère éminemment opportuniste des entrepreneurs innovants lui-même déterminé, et ceci est rarement souligné, par la spécificité de la TPE pour ce qui concerne les impulsions à l'innovation. Ce sont en effet les exigences des clients qui constituent pour elle la première impulsion, le «technology push» et la «demand pull» se situant derrière. Une déclinaison des deux impulsions traditionnelles de la théorie reprise par le manuel de Frascati aurait été, pour notre propos, lourd de contresens d'interprétation, car, 
comme nous l'avons montré ailleurs (Pacitto, 1996), la TPE ne connaît pas le marché, elle ne connaît que ses clients et ce n'est évidemment pas la même chose. Regrouper dans une même rubrique concurrence et clientèle eut été s'exposer à un risque de confusion certain, car ces deux réalités sont perçues d'une manière particulière et diffèrent profondément de la vision des moyennes et des grandes entreprises. Dans cette perspective, l'innovation n'est que rarement de nature proactive mais plutôt, comme le souligne Kaminski (1995), réactive, la TPE innove pour répondre à un besoin spécifique du client et rarement pour se positionner. On comprendra dès lors que la mobilisation des ressources soit elle-même opportuniste, donc que les modalités de cette mobilisation soient changeantes, même si certaines régularités apparaissent (Le Meun, 1993). On comprendra aussi que la notion d'apprentissage par le marché n'a que peu de sens pour la TPE et, de la même façon, immerger celle-ci dans des espaces concurrentiels jugés plus discriminants ne donnerait que peu de résultats.

Disposer de clients exigeants serait, par contre, plus profitable, car il y a un lien évident entre le nature des clients servis et la fréquence de l'innovation (Médus et Pacitto, 1994) ; ainsi, par exemple, les secteurs qui sont le plus en rapport avec les industries de la défense, l'aérospatiale ou le nucléaire et avec les grandes entreprises sont aussi ceux où l'on compte le plus d'entreprises innovantes.

\subsection{Une hypothèse : le rôle central du savoir-faire interne}

Ainsi, le savoir-faire interne constitue, pour la TPE, la première source de l'innovation. Il est significatif de relever que l'intensité moyenne du savoir-faire interne comme source d'innovation (2,02 tous secteurs confondus) est très largement supérieure à celle observée pour les autres sources de l'innovation. Cette primauté résulte d'une composante culturelle très forte observée au sein de la TPE et résidant dans l'attachement de celle-ci à la transmission du savoir-faire interne, du « coup de main » ; ce savoir-faire interne est reconnu, valorisé et transmis plus que protégé. Par ailleurs, deux observations importantes - qui ressortent de la simple lecture des résultats - méritent d'être formulées : la première source de l'innovation réside dans un bien incorporel peu ou pas protégé, souvent mobile et strictement attaché aux individus ; cela témoigne de la vulnérabilité des TPE à cristalliser durablement ce savoir-faire de l'entreprise, sauf à en organiser une transmission systématique... ou de la volonté / incapacité des TPE de ne pas durablement protéger ce savoir-faire. Dans le même temps, et ce constat renforce la primauté des sources internes, l'acquisition de valeurs incorporelles protégées (brevets et licences) constitue la source de l'innovation la plus négligeable, et ce, même au sein des entreprises fortement innovantes. De surcroît, la TPE adopte un comportement de rejet de la protection de son savoir-faire. À quasiment $70 \%$, les TPE se déclarent non concernées par le dépôt de brevets, ceux-ci étant jugés coûteux et inutiles. Il n'est pas sans intérêt de noter que les deux secteurs les plus innovants (biens professionnels 
et mécanique générale) sont aussi ceux où le savoir-faire interne obtient les scores les plus élevés. Pour ce qui concerne la TPE, l'appartenance sectorielle ne modifie pas de manière significative la distribution des sources de l'innovation. Quelle que soit l'intensité technologique du secteur, le savoir-faire interne reste la première source de l'innovation. Du point de vue de la théorie économique, la nature même de ce savoir n'est pas sans poser problèmes. Résumé par Polanyi (1966), le paradoxe du savoir-faire interne tient en une proposition simple: «nous pouvons faire plus que nous pouvons dire», autrement dit, si on peut décrire l'output, on est que peu renseigné sur la nature des inputs constitutifs du premier, ou, du moins, ceux-ci sont difficilement individualisables. C'est ce qu'a bien mis en évidence Rosenberg (1982), en montrant l'absence de lien entre les conséquences et l'incapacité constatée d'en expliquer les causes. Pour autant, le caractère tacite des savoirs n'est pas ignoré et fait même l'objet d'études serrées (Senker et Faulkner, 1996). Lorsqu'on envisage la question des savoirs tacites se pose bien évidemment le problème de leur origine.

Source de l'innovation, le savoir-faire interne est souvent lui-même la résultante «cumulative » des autres sources, en ce sens, sa catégorisation et son individualisation faussent quelque peu les perspectives. De plus, il est difficile de placer, dans le cas de la TPE, l'interaction compétitive comme un constituant potentiel de ce savoir (Barden et Good, 1989), plus sûrement et en suivant Gambardella (1992), il apparaît certain que celui-ci est largement alimenté par les réseaux personnels, souvent informels de l'entrepreneur (Steward et Conway, 1996). Dans ce cadre, l'élément fondamental à considérer sera la capacité de ce dernier à saisir l'information et à l'intégrer dans des savoirs déjà existants. S'il est difficile de mesurer cette capacité, on peut néanmoins tenter de la cerner. C'est ce que l'on a fait en construisant un indicateur synthétique d'ouverture sur l'extérieur. Il a été calculé à partir des différentes relations que l'entreprise entretient avec son environnement, en particulier les partenariats technologiques noués avec d'autres entreprises ou institutions. Il révèle donc la capacité à coopérer de ces entreprises ou, du moins, leur capacité à gérer ou à établir des relations interorganisationnelles souvent interpersonnelles. Même s'il faut rester prudent dans l'interprétation, il pourra nous renseigner sur l'effort informationnel des très petites entreprises et nous permettre d'entrevoir dans quels secteurs cette démarche est la plus marquée.

C'est le secteur le plus innovant qui obtient la meilleure moyenne. Ce sont les entreprises de ce secteur qui manifestent le plus grand souci informationnel et cela est confirmé par l'analyse de variance. On comprendra aisément que les pressions concurrentielles propres à ce secteur, de même que son fort contenu technologique, influent sur les comportements des chefs d'entreprise en facilitant et rendant indispensables les stratégies d'appropriation. Ainsi, il est intéressant de constater, et cela pour les autres relations "partenariales », que les entreprises les moins innovantes du secteur des biens professionnels ne sont pas moins coopératives que les plus innovantes, et c'est dans les secteurs les moins innovants que les différences sont 
Tableau 3

Ouverture sur l'extérieur des TPE

Structure dans chaque activité et moyenne générale de la variable

\begin{tabular}{lcccrrr}
\hline & \multicolumn{7}{c}{ Index d'ouverture en cinq positions } & & \\
& $\mathbf{1}$ & $\mathbf{2}$ & $\mathbf{3}$ & $\mathbf{4}$ & $\mathbf{5}$ & Moyenne \\
\hline Mécanique générale & 21,13 & 30,20 & 28,32 & 13,94 & 6,42 & 4,10 \\
Matériel agricole & 35,14 & 24,86 & 28,29 & 8,86 & 2,86 & 3,18 \\
Biens professionnels & 15,74 & 19,61 & 28,81 & 16,46 & 19,37 & 5,68 \\
Industries du bois & 14,12 & 29,58 & 38,74 & 12,98 & 4,58 & 4,19 \\
Textile - habillement & 33,84 & 30,51 & 26,59 & 1,81 & 7,25 & 3,24 \\
Biens de consommation & 27,60 & 31,15 & 25,68 & 8,47 & 7,10 & 3,67 \\
Installation électrique & 33,57 & 27,08 & 22,28 & 15,66 & 1,41 & 3,25 \\
Génie climatique & 33,59 & 29,14 & 25,94 & 8,02 & 1,60 & 2,94 \\
Toutes activités & 24,85 & 27,17 & 28,08 & 12,19 & 7,71 & 4,04 \\
\hline
\end{tabular}

$1^{\text {re }}$ colonne Indicateur au plus égal à 1 .

$2^{\mathrm{e}}$ colonne Indicateur compris entre 1,5 et 3 .

$3^{\mathrm{e}}$ colonne Indicateur compris entre 3,5 et 6,5 .

$4^{\mathrm{e}}$ colonne Indicateur compris entre 7 et 9,5 .

$5^{\mathrm{e}}$ colonne Indicateur au moins égal à 10.

Source : Enquête TIME.

le plus marquées; les entreprises les moins innovantes de ces secteurs étant plus « coopératives » que les moins innovantes. Néanmoins est ici donnée confirmation de la stratégie de captation que développent les entreprises les plus innovantes pour accroître leurs ressources et par là même leur savoir-faire. Il faut par ailleurs relativiser « l'informalité » des réseaux des petites entreprises ; les résultats de l'enquête montrent en effet que les partenaires institutionnels sont présents, mais que l'importance de cette présence dépend en dernier lieu de la structuration de chacun des secteurs, notamment de leur structuration professionnelle.

\subsection{La vraie place des activités de R-D}

Au-delà des premiers constats, il nous est apparu nécessaire de comparer la distribution des sources d'innovation mais, cette fois-ci, en différenciant, les très petites entreprises innovantes des moins innovantes. La question sous-jacente était de savoir si on n'allait pas retrouver dans ce cas, et pour les entreprises les plus innovantes, une distribution des sources proche de celles de la moyenne et grande entreprise, notamment en ce qui concerne la recherche-développement.

De fait, en opérant cette distinction, on obtient un résultat proche de celui des moyennes et grandes entreprises, surtout en ce qui concerne la recherchedéveloppement. Celle-ci devient pour les TPE les plus innovantes la deuxième source 
TABLEAU 4

Sources de l'innovation dans les entreprises moins innovantes et dans les plus innovantes

\begin{tabular}{|c|c|c|c|c|c|c|c|c|c|c|}
\hline Secteurs & Entreprises & S1 & $\mathbf{S 2}$ & S3 & S4 & S5 & S6 & S7 & S8 & S9 \\
\hline \multirow[t]{2}{*}{ MECA } & $\begin{array}{l}\text { Moins } \\
\text { innovantes }\end{array}$ & 0,33 & 1,70 & 0,20 & 0,06 & 0,46 & 0,85 & 1,56 & 0,39 & 0,36 \\
\hline & $\begin{array}{l}\text { Plus } \\
\text { innovantes }\end{array}$ & 1,33 & 3,36 & 0,84 & 0,22 & 1,05 & 1,45 & 1,98 & 0,99 & 0,93 \\
\hline \multirow[t]{2}{*}{ AGRI } & $\begin{array}{l}\text { Moins } \\
\text { innovantes }\end{array}$ & 0,40 & 1,68 & 0,08 & 0,05 & 0,36 & 0,49 & 0,30 & 0,12 & 0,15 \\
\hline & $\begin{array}{l}\text { Plus } \\
\text { innovantes }\end{array}$ & 1,79 & 3,29 & 1,40 & 1,07 & 0,32 & 1,53 & 1,61 & 1,47 & 0,75 \\
\hline \multirow[t]{2}{*}{ PROF } & $\begin{array}{l}\text { Moins } \\
\text { innovantes }\end{array}$ & 0,53 & 1,55 & 0,14 & 0,03 & 0,35 & 0,83 & 0,90 & 0,38 & 0,52 \\
\hline & $\begin{array}{l}\text { Plus } \\
\text { innovantes }\end{array}$ & 2,17 & 3,57 & 0,61 & 0,25 & 0,74 & 1,47 & 1,75 & 1,38 & 2,05 \\
\hline \multirow[t]{2}{*}{ BOIS } & $\begin{array}{l}\text { Moins } \\
\text { innovantes }\end{array}$ & 0,28 & 1,53 & 0,17 & 0,04 & 0,34 & 0,49 & 0,76 & 0,28 & 0,40 \\
\hline & $\begin{array}{l}\text { Plus } \\
\text { innovantes }\end{array}$ & 1,34 & 2,14 & 0,59 & 0,19 & 0,90 & 1,67 & 1,69 & 1,36 & 1,34 \\
\hline \multirow[t]{2}{*}{ TEXT } & $\begin{array}{l}\text { Moins } \\
\text { innovantes }\end{array}$ & 0,01 & 2,24 & 0,02 & 0,00 & 0,38 & 0,19 & 1,98 & 0,02 & 0,52 \\
\hline & $\begin{array}{l}\text { Plus } \\
\text { innovantes }\end{array}$ & 0,83 & 3,04 & 1,51 & 0,25 & 1,13 & 1,54 & 2,27 & 1,39 & 1,53 \\
\hline \multirow[t]{2}{*}{ MENA } & $\begin{array}{l}\text { Moins } \\
\text { innovantes }\end{array}$ & 0,08 & 1,14 & 0,27 & 0,00 & 0,44 & 0,34 & 0,51 & 0,44 & 0,55 \\
\hline & $\begin{array}{l}\text { Plus } \\
\text { innovantes }\end{array}$ & 1,51 & 3,44 & 0,90 & 0,50 & 1,53 & 1,95 & 1,52 & 0,67 & 2,01 \\
\hline \multirow[t]{2}{*}{ ELEC } & $\begin{array}{l}\text { Moins } \\
\text { innovantes }\end{array}$ & 0,26 & 1,85 & 0,12 & 0,00 & 0,54 & 0,58 & 0,29 & 0,56 & 1,43 \\
\hline & $\begin{array}{l}\text { Plus } \\
\text { innovantes }\end{array}$ & 1,04 & 2,59 & 1,01 & 0,01 & 1,29 & 1,47 & 0,71 & 0,70 & 1,97 \\
\hline \multirow[t]{2}{*}{ CLIM } & $\begin{array}{l}\text { Moins } \\
\text { innovantes }\end{array}$ & 0,09 & 1,28 & 0,17 & 0,01 & 0,66 & 0,53 & 0,37 & 0,21 & 0,79 \\
\hline & $\begin{array}{l}\text { Plus } \\
\text { innovantes }\end{array}$ & 1,09 & 3,41 & 0,70 & 0,02 & 1,21 & 1,45 & 0,88 & 1,63 & 2,49 \\
\hline \multirow[t]{2}{*}{ TOTAL } & $\begin{array}{l}\text { Moins } \\
\text { innovantes }\end{array}$ & 0,31 & 1,64 & 0,16 & 0,04 & 0,41 & 0,65 & 1,16 & 0,32 & 0,48 \\
\hline & $\begin{array}{l}\text { Plus } \\
\text { innovantes }\end{array}$ & 1,58 & 3,25 & 0,83 & 0,26 & 0,95 & 1,52 & 1,56 & 1,18 & 1,47 \\
\hline
\end{tabular}

Source : Enquête TIME. 
de l'innovation, devançant les nouvelles machines et les nouveaux matériaux, alors qu'elle n'est que la septième source pour les TPE les moins innovantes. On remarquera aussi que les nouveaux matériaux ne constituent plus que la cinquième source, après les nouvelles machines; ce qui est aussi le cas des PME où l'on a vu que les machines devancent les nouveaux matériaux. On ne sera pas surpris, de même, de constater que ce sont les entreprises les plus innovantes du secteur le plus innovant qui effectuent le plus de recherche-développement. La liaison input / output est dans ce cas vérifiée, les très petites entreprises les plus innovantes pratiquent la recherchedéveloppement et celle-ci constitue une source majeure d'innovation. On n'est sûrement pas en présence d'une activité « continue » et « organisée » mais, dans tous les cas, d'un effort conscient et soutenu en vue du développement des produits, des techniques de fabrication ou de la recherche de nouveaux produits. Pour autant, les processus informels de recherche de l'information ne sont pas négligés, ce qui démontre l'extrême difficulté qu'éprouve la TPE à dissocier R-D formelle et R-D informelle (Santarelli et Sterlacchini, 1990). De toute façon, la nature même des processus organisationnels à l'œuvre dans la très petite entreprise interdirait toute rationalisation de l'activité $\mathrm{R}-\mathrm{D}$, et il ne s'agit pas ici seulement de phénomènes de bureaucratisation (Scherer, 1991). La R-D est donc en dernier lieu une réalité pour la TPE innovante, c'est simplement sa structuration au sein de la structure qui diffère, même si on peut s'interroger sur la pertinence du modèle linéaire pour la grande entreprise (Smith, 1989). Contrairement à une opinion trop souvent avancée, la TPE innovante ne compense pas son manque de recherche-développement par un recours plus marqué à l'apprentissage par l'interaction. En premier lieu, parce que, comme on l'a vu, la R-D constitue une source majeure d'innovation et, en second lieu, parce que les deux phénomènes sont liés, complémentaires.

Le problème de la production du savoir n'est pas pour autant résolu mais une chose apparaît certaine : la capacité opportuniste des entrepreneurs est liée à leurs ressources ; il ne peut dès lors y avoir compensation (Rizzoni, 1994) ; ce sont ces ressources qui permettent à l'entreprise d'exploiter au mieux les sources d'innovation et ainsi de transformer les divers inputs en outputs. Dans cette perspective, et nous rejoignons ici Audretsch et Vivarelli, il importe de bien saisir les mécanismes qui permettent au chef d'entreprise d'intégrer ces savoirs, même si leur caractère tacite et les aspects cognitifs de ces processus rendent difficiles une possible description ou pour le moins une théorisation. Si les mécanismes de transformation des ressources s'avèrent difficiles à appréhender, il n'en demeure pas moins que certaines d'entre elles apparaissent comme déterminantes. Ainsi la capacité à innover est clairement corrélée, dans le cas de la très petite entreprise, avec le niveau de formation des entrepreneurs : c'est elle qui détermine le degré de sensibilité à l'innovation. Il ne servirait à rien pour une TPE de multiplier ses contacts si, dans un même temps, elle s'avère incapable d'exploiter les informations qui y circulent (Gambardella, 1992). De la même façon, la recherche-développement constitue pour 
les très petites entreprises innovantes non une ressource de base mais plutôt un générateur de ressources qui va permettre d'intégrer des connaissances acquises par le canal des autres sources. C'est en cela que la TPE innovante se distingue des autres ; il ne s'agit pas ici de linéarité des processus mais de capacité à intégrer ou, selon l'expression de Pavitt, à s'approprier, cette appropriation dépendant en dernière instance de la capacité d'absorption de l'entreprise (Cohen et Levinthal, 1989). On nous objectera que ceci se vérifie aussi dans le cadre des grandes entreprises, simplement pour la très petite entreprise, ces fonctions sont souvent assumées par le même homme, ce qui modifie radicalement les données du problème et la nature du processus de transformation des inputs en outputs. Dans une même perspective, Johanninson (1993) et Kim (1993) ont pu établir qu'au-delà des phénomènes d'apprentissage de type opérationnel, ce sont bien les connaissances conceptuelles et leur élargissement qui permettent aux entreprises d'acquérir des avantages concurrentiels. En effet, c'est à partir de ces connaissances que l'entreprise pourra tirer des avantages de la compréhension et de la traduction des expériences passées en nouvelles initiatives. De plus, et comme le souligne Quelin (1996), il importe de bien comprendre que les ressources d'une entreprise sont totalement liées aux capacités de combinaison, d'exploitation et de conversion, et on peut émettre l'hypothèse que celles-ci sont aussi liées au niveau de recherche-développement dans l'entreprise.

\subsection{Les TPE et le dépôt de brevets}

Il serait réducteur de ne vouloir considérer les brevets que comme un seul indicateur d'innovation. Comme Griliches $(1984,1990)$ a tenté de le montrer, les brevets sont aussi un indicateur de la performance économique. Toutefois, l'utilisation des brevets comme mesure de la performance à l'innovation n'est pas sans poser problème (Archibugi et Pianta, 1994).

Cette difficulté est aggravée quand on examine la relation entre la taille des entreprises et le dépôt de brevets (Le Bas, 1995). Les résultats sont souvent contradictoires et donc difficiles à interpréter.

De la même façon, la « sectorialisation » du phénomène (Scherer, 1982) n'a pas apporté de résultats décisifs. La difficulté à interpréter provient aussi des méthodologies souvent très différentes utilisées dans les enquêtes d'innovation (Le Bas et Torré, 1993). En ce qui concerne la très petite entreprise, les données sont quasiment inexistantes, les dernières enquêtes innovation (Service des statistiques industrielles, 1994) ne prenant, en France, pas en compte les entreprises de moins de 20 salariés. De fait, il apparaissait difficile de répondre à la question simple : les TPE déposent-elles des brevets ? Parmi les TPE, 10,3\% déposent des brevets ou détiennent des droits de propriété industrielle, ce qui peut apparaître faible dans l'absolu ou relativement fort si l'on compare ce pourcentage à celui des entreprises innovantes ( $20 \%$ d'entreprises innovantes, tous secteurs confondus). 
En prolongeant cette réflexion par l'analyse du lien entre la taille de l'entreprise et le dépôt de brevets, on observe que les TPE s'inscrivent parfaitement dans les tendances générales concernant la liaison taille et dépôts de brevets.

\section{TABLEAU 5}

\section{Pourcentage d'entreprises déposant des brevets par effectifs de salariés}

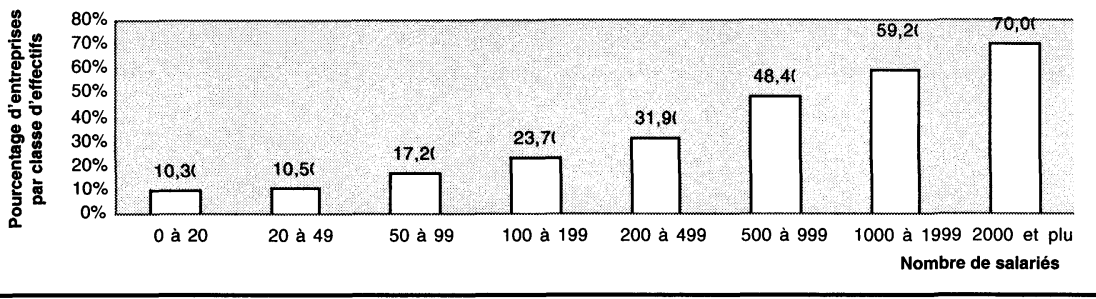

Source : Enquête TIME pour les moins de 20 salariés.

EAE 90 pour les entreprises industrielles de plus de 20 salariés

Si l'on s'intéresse aux déterminants, on constate que la propension à déposer des brevets est clairement corrélée avec deux facteurs :

- l'appartenance à un secteur de métier ;

- le niveau de formation existant dans l'entreprise.

Plus du quart des entreprises de fabrication de biens professionnels déclarent avoir déposé au moins un brevet alors que ce pourcentage est marginal pour les entreprises spécialisées dans la réparation électrique ou le génie climatique.

\section{TABLEAU 6}

\section{Pourcentage des TPE déposant des brevets par secteur d'activité}

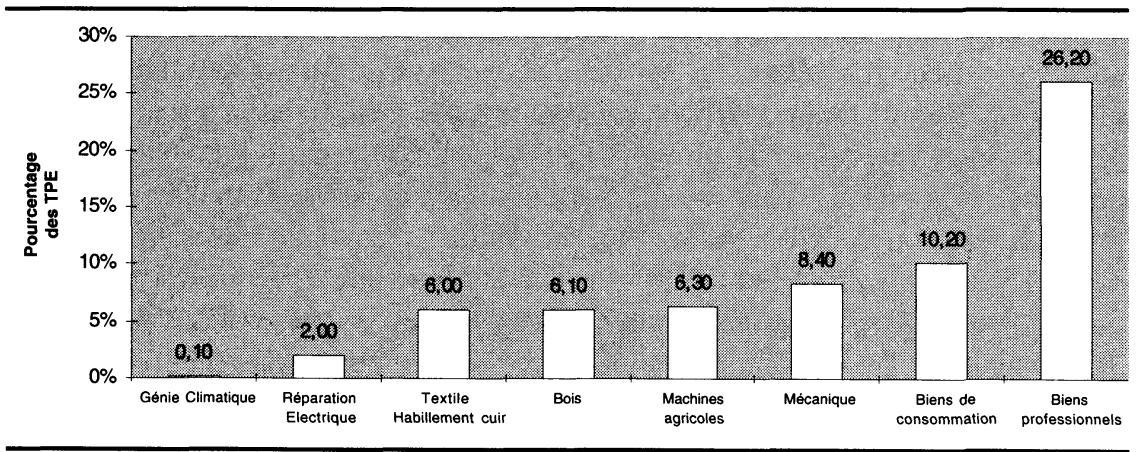


Il est ici intéressant de constater que le «palmarès » des «meilleurs breveteurs » ne coïncide pas parfaitement avec celui des champions de l'innovation. Ainsi, si les biens professionnels occupent bien la première place de ce classement, la mécanique n'arrive qu'en troisième position derrière les biens de consommation. Ce qui doit s'expliquer par l'importance relative du deuxième déterminant : le niveau de formation du personnel.

Alors que 10,3\% seulement des TPE déposent des brevets, le quart des entreprises où au moins l'un des collaborateurs bénéficie d'un niveau de formation d'école d'ingénieur ou d'université déposent des brevets.
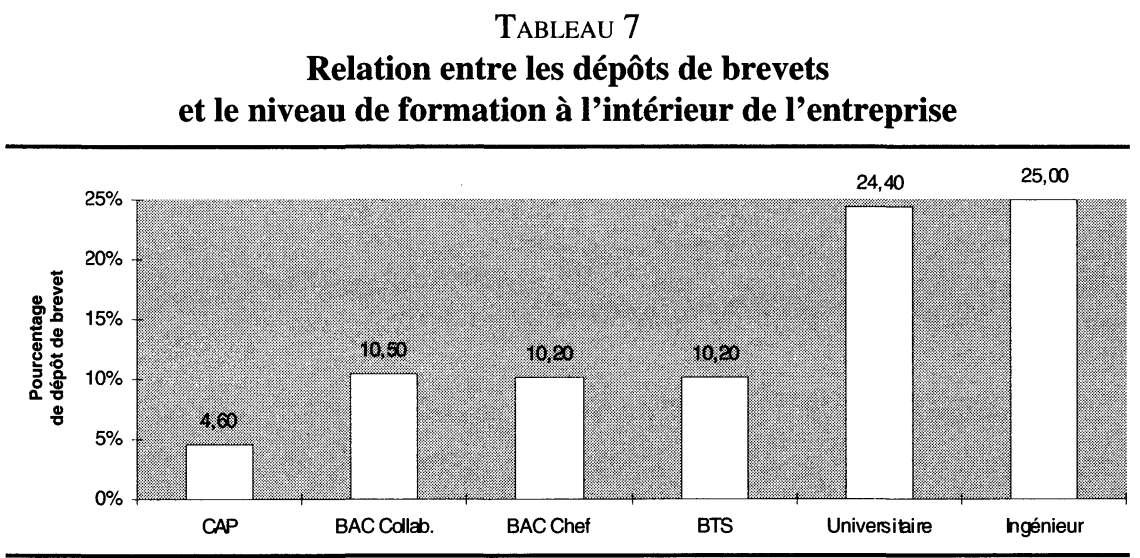

Source : Enquête TIME.

Celles dont les collaborateurs et le chef d'entreprise n'ont qu'une formation professionnelle en déposent le moins, et enfin, les formations secondaires ou de techniciens supérieurs se situent autour de la moyenne. On explique donc en partie ici les bonnes performances du secteur des biens de consommation où le niveau de qualification est le plus élevé après celui des biens professionnels. De la même manière, la «modeste » performance des entreprises de mécanique peut s'analyser comme un manque relatif de formation du dirigeant ou des collaborateurs. Si plus de la moitié des TPE n'expriment pas d'opinion sur les dépôts de brevet (52\%), force est de constater qu' une part importante des chefs d'entreprises les juge coûteux $(26,0 \%)$ ou inutiles $(21,9 \%)$. Il peut paraître à première vue surprenant que les jugements les plus défavorables soient portés par des responsables d'entreprises qui évoluent dans les secteurs brevetant le plus. On émettra ici l'hypothèse que ces dirigeants ont, plus fréquemment que d'autres, envisagé une protection juridique de leurs innovations et qu'ils en mesurent mieux le coût réel. Un point de vue qui est conforté par le fait que ce sont précisément les plus diplômés qui jugent le plus fréquemment les dépôts trop coûteux. 
TABLEAU 8

Freins au dépôt de brevets

\begin{tabular}{lccc}
\hline & Coûteux & Inutile & Ne sait pas \\
\hline Ensemble & $\mathbf{2 6}$ & $\mathbf{2 1 , 9}$ & $\mathbf{5 2 , 1}$ \\
\hline Activité & & & \\
Mécanique générale & 27,5 & 22,6 & 49,9 \\
Matériel agricole & 24,9 & 18,9 & 56,3 \\
Biens professionnels & 36,7 & 27,5 & 35,8 \\
Industries du bois & 21,8 & 21,4 & 56,9 \\
Textile-habillement & 12,7 & 40,8 & 46,5 \\
Biens de consommation & 26,2 & 18,4 & 55,3 \\
Installation électrique & 23,4 & 7,8 & 68,8 \\
Génie climatique & 15,5 & 24,6 & 59,9 \\
\hline Formation & & & \\
CAP & 19,1 & 19,9 & 61,0 \\
BAC « Dirigeant » & 27,1 & 22,3 & 50,6 \\
BAC «Collaborateur » & 24,5 & 27,4 & 48,1 \\
BTS & 34,1 & 20,7 & 45,2 \\
FAC & 36,3 & 23,6 & 40,1 \\
INGEN & 33,3 & 25,2 & 41,6 \\
\hline
\end{tabular}

Source : Enquête TIME.

On remarquera aussi que les secteurs où les dépôts de brevets sont les plus nombreux correspondent aussi à ceux où les phénomènes de coopération sont le plus marqués (tableau 3). Cependant, la prudence s'impose quant à l'interprétation à donner à ces résultats, rien ne permet dans l'enquête de conclure ainsi à un rôle particulier joué par les laboratoires universitaires ou autres. De surcroît, les causalités s'enchevêtrent et il est bien évident, comme l'a montré Rizzoni (1994), que la capacité à coopérer dépend des ressources possédées par l'entreprise et que, dans le cas de la très petite entreprise, le niveau de formation est une ressource fondamentale (Zagouras et Papanayotou, 1995).

\section{Les réseaux, une caractéristique distinctive ?}

Devenu facteur de compétitivité, la relation de l'entreprise avec l'extérieur et surtout les contacts qu'elle noue et entretient avec les différents éléments de son environnement ont fait l'objet, ces dernières années, de nombreuses études souvent rassemblées dans ce mode particulier de coopération interentreprise que constitue le réseau (Paché et Paraponaris, 1993). 
De la sorte et inévitablement, la capacité de la firme à nouer des relations avec son environnement devient, comme le montre Rothwell (1994), un facteur favorisant l'innovation, mais aussi par là une source de l'innovation, même si le passage de l'un à l'autre n'apparaît pas évident. Le problème de l'analyse en termes de réseau tient, pour une bonne part, à un problème de définition, et au fond, qu'est-ce qui permet à un moment donné de qualifier les liens que noue l'entreprise avec ses différents partenaires de réseau ? L'extrême variété des phénomènes observés appelle, dans un premier temps, une définition large et c'est, dans un second temps seulement - dans la qualification des particularités -, que pourront être proposées des typologies plus restrictives. Il ne nous appartient pas ici de discuter une très nombreuse littérature et nous reprendrons une définition proposée par Bouvier-Patron (1996) qui voit le réseau « comme un ensemble d'acteurs dont la coordination n'est plus strictement marchande et dont la finalité est la conception, la production et la vente de produits».

Cette définition est proche de celle proposée par Axelson et Easton (1992), où le réseau apparaît comme un modèle ou une métaphore qui décrit un grand nombre d'entités liées entre elles, impliquées dans des processus économiques qui convertissent des ressources en biens et services propres à la consommation des clients finaux, individus ou organisations. Poser le problème des réseaux et de leur rôle dans l'innovation, c'est aussi immanquablement évoquer le problème de l'apprentissage. Dodgson (1996) montre bien qu'à travers ces réseaux, les entreprises essaient de franchir des paliers au regard de savoir-faire technologique, de compenser pour des ressources internes insuffisantes mais aussi de faire évoluer leur culture d'entreprise (Argyris et Schön, 1978; Minguzzi et Passaro, 1995). Quelin (1996), dans une perspective plus générale, insiste sur la nécessité pour les entreprises de créer des nouvelles ressources et d'étendre leurs capacités à en générer. C'est dans cette optique que, selon lui, doivent être réexaminées les coopérations interentreprises. Car il ne faudrait pas oublier, et cela a été mis en évidence par Rizzoni (1994), que l'ampleur des coopérations dépendra en grande partie des ressources possédées et, comme l'ont aussi montré Teece (1988), Amendola et Gaffard (1988), la capacité d'innovation de la firme et la création de ressources nouvelles reposent sur l'existence d'un fond de compétences centrales accumulées et valorisées par la firme. On comprendra que le réseau ne peut être dès lors l'espèce de panacée universelle visant à transformer les entreprises (CGP, 1993) mais un phénomène, quoi qu'on en dise, contingent, parce que lié précisément à la nature des ressources possédées par les entreprises ou, pour parler comme Williamson, aux types d'actif. De surcroît, le développement des réseaux tient, pour ce qui concerne la petite entreprise, à son mode particulier d'apprentissage.

Ce mode d'apprentissage, comme le souligne Le Meun (1993), se caractérise par un perfectionnement fondé sur la capitalisation de l'expérience quotidienne allié aux contacts de réseaux d'acteurs privilégiés. Ainsi, note le même auteur, «les 
connaissances personnelles du dirigeant, les lieux qu'il fréquente ou qu'il a fréquentés sont des déterminants fondamentaux du fonctionnement de l'apprentissage ». L'apprentissage par l'interaction n'est pas, pour la TPE, un mode d'apprentissage parmi d'autres mais le mode d'apprentissage privilégié. Ces réseaux d'acteurs sont le plus souvent issus non d'un choix libre mais d'un choix largement déterminé par les réseaux de socialisation du dirigeant parmi lesquels, comme l'a montré Perrien (1994), il sélectionnera ses nouvelles relations. C'est d'une certaine manière ce que suggère Pecqueur (1989) en délimitant pour la TPE deux niveaux de structuration de ces réseaux.

Un premier niveau, qualifié de réseaux à finalité productive, qui comprend tous les acteurs avec lesquels l'entreprise compose habituellement, et un deuxième niveau, qualifié de réseaux informels, qui regroupe tous les réseaux de nature familiale, professionnelle et institutionnelle. Cette délimitation nous semble judicieuse même s'il nous semble, en accord avec Lecoq (1989), qu'il faille individualiser la dernière catégorie (réseaux institutionnels), car elle nous paraît relever d'un type relationnel particulier, loin du qualificatif «informel». Si l'on admet que la démarche innovatrice nécessite un surcroît d'informations, il nous appartiendra de vérifier cette assertion. Peut-on mettre au jour, dans le cas de la TPE innovante, une démarche informationnelle plus prononcée que pour la moins innovante qui se traduirait notamment par un recours plus systématique aux réseaux ? Ceux-ci constitueraient-ils dès lors une caractéristique distinctive de la TPE innovante ? Nous nous intéresserons, ici, plus particulièrement mais pas exclusivement, aux partenaires «non naturels », ceux avec qui la TPE ne compose pas naturellement, c'est-à-dire ceux qui vont nécessiter de sa part une démarche spécifique, démarche qui traduira sa capacité à capter des ressources et, ce faisant, à coopérer, la coopération étant, comme l'écrit justement De Bandt (1996), « une conjugaison de savoirs et d'efforts en vue de réaliser quelque chose ». Les résultats de l'enquête apportent sur ces différentes interrogations un certain nombre d'éclaircissements mais il faut rester prudent quant à l'interprétation, car, en dernier lieu, savons-nous réellement distinguer ce qui tient de la cause et ce qui tient de l'effet? Les réseaux existent mais leur contribution exacte au processus d'innovation reste, quoi qu'on en dise, difficilement évaluable et nous devons plus que par le recours à des assertions trop facilement répétées, avancer sur le mode de l'hypothétique.

\subsection{La recherche de l'information}

\section{Information organisée, information informelle (tableau 4).}

Souvent affirmée (CGP, 1994), la relation entre innovation et information se vérifiet-elle pour autant empiriquement ? Si l'on agrège les informations organisées (salons professionnels) et les informations informelles, l'acquisition d'informations constitue une source majeure de l'innovation, et ce, tous secteurs confondus. Cette 
acquisition d'informations passe par de multiples canaux ; malgré son caractère éclaté, on est bien en présence, comme on l'a déjà souligné, d'un processus cumulatif où les effets de boucle entre les informations (acquisition, exploitation) sont mis en évidence (Kline et Rosenberg, 1986). On notera avec intérêt que, pour les entreprises les plus innovantes, l'information, qu'elle soit formelle ou informelle, obtient des scores partout supérieurs à ceux des entreprises les moins innovantes. La quasi absence de R-D pour ces dernières n'est donc pas compensée par un recours plus marqué à l'information, et cela, quelle que soit sa provenance. Le recours et la sensibilisation à l'information apparaissent bien comme des traits distinctifs des TPE les plus innovantes et, à cet égard l'influence sectorielle n'est que peu discriminante.

\subsection{Information documentaire et partenariats}

TABleau 9

Entreprises, information et partenariats (chiffres pondérés)

\begin{tabular}{llrrrc}
\hline & Entreprises & Part 1 & Part 2 & Part 3 & Part 4 \\
\hline Ensemble & Les moins innovantes & 19,08 & 72,28 & 50,28 & \multicolumn{1}{c}{26,7} \\
& Les plus innovantes & 35,11 & 87,49 & 77,12 & 43,93 \\
\hline Activité & & & & & \\
Mécanique & Les moins innovantes & 25,67 & 77,59 & 57,74 & 30,38 \\
générale & Les plus innovantes & 40,70 & 83,96 & 88,32 & 42,24 \\
Matériel & Les moins innovantes & 14,84 & 86,43 & 50,84 & 20,16 \\
agricole & Les plus innovantes & 33,10 & 75,28 & 70,35 & 20,70 \\
Biens & Les moins innovantes & 23,52 & 78,51 & 68,53 & 27,80 \\
professionnels & Les plus innovantes & 37,32 & 91,95 & 74,94 & 45,40 \\
Industries & Les moins innovantes & 15,36 & 82,50 & 39,40 & 21,16 \\
du bois & Les plus innovantes & 32,67 & 88,27 & 68,06 & 46,71 \\
Textile- & Les moins innovantes & 2,56 & 22,01 & 22,21 & 26,15 \\
habillement & Les plus innovantes & 1,07 & 85,33 & 62,60 & 45,26 \\
Biens de & Les moins innovantes & 11,66 & 79,67 & 33,62 & 20,98 \\
consommation & Les plus innovantes & 40,17 & 87,84 & 63,20 & 45,92 \\
Installation & Les moins innovantes & 26,36 & 73,09 & 46,70 & 34,37 \\
électrique & Les plus innovantes & 60,14 & 100,00 & 81,99 & 76,73 \\
Génie & Les moins innovantes & 5,14 & 87,31 & 43,46 & 15,32 \\
climatique & Les plus innovantes & 47,93 & 93,29 & 61,16 & 17,44 \\
\hline
\end{tabular}

Part 1 Abonnement à une revue en informatique.

Part 2 Abonnement à une revue technique spécialisée.

Part 3 Abonnement à une revue technique générale.

Part 4 Partenariat technique avec une autre entreprise.

Source : Enquête TIME. 
Les TPE les plus innovantes manifestent un souci documentaire plus grand que leurs homologues moins innovantes, qu'il s'agisse des abonnements à des revues techniques générales ou à des revues techniques spécialisées.

À part le cas du secteur du textile-habillement, l'augmentation du score lorsqu'on passe des entreprises les moins innovantes aux plus innovantes et pour ce qui concerne l'abonnement à une revue technique spécialisée demeure relatif. C'est surtout pour la rubrique abonnement à une revue technique générale que la différence est manifeste. Ceci tendrait à démontrer de la part de ces entreprises une curiosité plus grande par rapport à l'environnement et une volonté plus affirmée d'en comprendre les évolutions générales. Dans un même ordre d'idées, il n'est pas sans importance de constater que les entreprises les plus innovantes éprouvent, par rapport à l'informatique, une curiosité nettement plus marquée que les entreprises les moins innovantes. Au-delà du seul aspect technique, l'informatique et la sensibilisation à ce domaine apparaissent comme des révélateurs d'une certaine veille technologique et, plus globalement, environnementale. L'intérêt qu'y portent les TPE les plus innovantes contraste avec la quasi-indifférence des TPE les moins innovantes, de surcroît, quand ce thème devient omniprésent. La recherche de l'information pertinente et plus globalement de la ressource informationnelle passe aussi par la relation avec les autres entreprises. On sait (Marchesnay, 1992 ; Pacitto, 1996) que les TPE sont réticentes aux démarches partenariales conjointes et que la notion de projet individuel l'emporte souvent sur la nécessité, même ressentie, de coopérer. Toutefois, ce premier constat doit être nuancé, car là aussi la comparaison des TPE moins innovantes et des TPE plus innovantes laisse apparaitre des résultats intéressants en ce sens que le partenariat technique avec une autre entreprise (PME ou autres) semble être une caractéristique distinctive des TPE les plus innovantes. Celles-ci n'hésitent pas à nouer des relations avec d'autres entreprises et ne considèrent pas la coopération comme une perte d'indépendance. La recherche de l'information pertinente, et par là même d'un surcroit de compétitivité, l'emporte sur des considérations d'ordre plus socioculturel.

De la sorte, l'impératif économique, par le biais technologique, estompe les réticences liées à un système de valeurs particulier où les attitudes découlant de l'individualisme sont survalorisées. Il s'agit, assurément, d'un moyen pour la TPE de se connecter avec un environnement plus incertain parce que plus technologique. Toutefois, si cette orientation est acceptée, elle n'est pas véritablement intériorisée, les TPE, qu'elles soient innovantes ou non, rejettent l'idée même de réseau.

\subsection{Relations institutionnelles et R-D sous-traitée}

Une des principales faiblesses de la TPE résulte sans aucun doute de son faible accès aux organismes officiels en charge du transfert technologique et, notamment, de l'ANVAR. 
Information incomplète, manque de temps, caractère trop technique et administratif des dossiers, les raisons ne manquent pas pour expliquer cet état de fait. Ainsi, malgré des efforts certains en direction de la TPE, l'ANVAR continue à surprivilégier les PME de plus de 50 salariés (INSEE, ANVAR). De la même façon, on observe une réticence de la part de ces entreprises à externaliser, surtout lorsque cela touche au cœur du savoir-faire, comme par exemple la recherche-développement. Pourtant, là aussi, la comparaison entre TPE moins innovantes et TPE plus innovantes montre des réalités différentes, des profils distinctifs.

\section{TABLEAU 10}

Entreprises, partenariats et R-D sous-traitée

\begin{tabular}{llrrrrrr}
\hline & Entreprises & Part 1 & Part 2 & Part 3 & Part 4 & Part 5 & Part 6 \\
\hline Ensemble & Moins innovantes & 7,40 & 19,91 & 4,83 & 4,89 & 0,87 & 0,79 \\
& Plus innovantes & 17,28 & 23,69 & 9,79 & 12,26 & 2,97 & 2,31 \\
\hline Activité & & & & & & & \\
Mécanique & Moins innovantes & 5,85 & 18,43 & 4,32 & 6,73 & 0,83 & 0,00 \\
générale & Plus innovantes & 8,97 & 19,12 & 7,27 & 10,86 & 0,00 & 0,00 \\
Matériel & Moins innovantes & 1,52 & 17,85 & 0,00 & 0,43 & 0,00 & 0,00 \\
agricole & Plus innovantes & 31,03 & 1,72 & 16,20 & 27,41 & 0,00 & 0,00 \\
Biens & Moins innovantes & 21,19 & 26,30 & 6,67 & 6,47 & 2,21 & 0,00 \\
professionnels & Plus innovantes & 20,40 & 28,61 & 15,53 & 11,30 & 2,96 & 6,87 \\
Industries & Moins innovantes & 2,70 & 24,30 & 7,06 & 1,31 & 0,00 & 0,00 \\
du bois & Plus innovantes & 15,98 & 37,13 & 8,28 & 2,76 & 16,55 & 0,00 \\
Textile- & Moins innovantes & 0,00 & 15,51 & 0,68 & 1,74 & 0,00 & 4,55 \\
habillement & Plus innovantes & 68,49 & 20,01 & 0,15 & 26,99 & 0,00 & 0,00 \\
Biens de & Moins innovantes & 6,33 & 18,61 & 2,90 & 6,67 & 0,95 & 1,22 \\
consommation & Plus innovantes & 17,62 & 26,13 & 13,95 & 8,91 & 3,93 & 2,25 \\
Installation & Moins innovantes & 1,13 & 6,15 & 10,91 & 3,19 & 0,03 & 3,98 \\
électrique & Plus innovantes & 0,00 & 13,44 & 4,42 & 17,80 & 1,32 & 0,00 \\
Génie & Moins innovantes & 0,00 & 8,54 & 7,44 & 9,27 & 2,63 & 0,00 \\
climatique & Plus innovantes & 18,78 & 6,71 & 0,00 & 11,18 & 0,00 & 4,02 \\
\hline
\end{tabular}

Part 1 Relation avec l'ANVAR.

Part 2 Relation avec un autre partenaire institutionnel.

Part 3 RD sous-traitée à une autre entreprise.

Part 4 RD sous-traitée à une société de service.

Part 5 RD sous-traitée à un organisme professionnel.

Part 6 RD sous-traitée à un laboratoire public.

Source : Enquête TIME.

Pour ce qui concerne les relations avec l'ANVAR, il faut rester prudent quant à l'interprétation à donner aux résultats. En effet, on remarque que les plus fortes variations concernent uniquement les secteurs traditionnellement les moins 
innovants (matériel agricole, textile-habillement, industries du bois), pour les secteurs les plus innovants (mécanique générale, biens professionnels), les variations sont faibles voire négatives (biens professionnels). L'ANVAR paraît constituer, pour les entreprises évoluant dans des secteurs technologiquement moins porteurs, une ressource importante, en tous les cas, un point de passage non négligé, ce qui n'est pas le cas pour les entreprises appartenant à des secteurs à plus forte intensité technologique.

Les mêmes remarques peuvent être faites au regard des relations avec les autres partenaires institutionnels (organisations professionnelles, centres techniques, etc.); la différenciation entre entreprises moins innovantes et entreprises plus innovantes s'opère principalement dans le cadre des secteurs traditionnels. Les entreprises les plus innovantes des secteurs les plus technologiquement porteurs n'ont pas, à ce niveau, un comportement sensiblement différent de celles moins innovantes. Ces résultats font ressortir l'influence du secteur d'activité sur les trajectoires des entreprises; ainsi, on a bien, pour les entreprises des secteurs les plus innovants, un type de trajectoire à la fois technologique et comportemental qui ne varie pas forcément avec l'accroissement de la capacité technologique. De la sorte, il serait prématuré et d'une certaine manière erroné de conclure à une attitude plus « réticulaire » de ces entreprises, la configuration particulière des secteurs déterminant bien davantage certains types de trajectoires. Les enjeux stratégiques propres à ces secteurs induisent un mode particulier de structuration des entreprises à l'environnement qui n'est pas sensible ou qui l'est peu à la montée en puissance technologique des entreprises le composant. Trop d'études tendent à négliger cet acquis et à généraliser un type de comportement, et cela, tous secteurs confondus. La relation partenariale n'apparaît comme un atout, une ressource que pour les entreprises des secteurs les moins innovants; elle apparaît clairement dans ce cadre comme une variable discriminante. Pour les autres secteurs d'activité, il faut nuancer les conclusions qu'une moyenne générale tendrait à induire. Négligeable pour ce qui touche la relation institutionnelle, la variable comportementale joue, par contre, à fond dans le cas de la R-D sous-traitée. Si l'on écarte le cas particulier de la R-D sous-traitée à un organisme professionnel et des secteurs particuliers (génie climatique et textile-habillement / main-d'œuvre sous-traitée à une autre entreprise), les entreprises les plus innovantes, et ce, tous secteurs confondus, obtiennent des scores partout supérieurs à ceux des entreprises les moins innovantes. On notera également, dans ce domaine, le faible rôle joué par les organismes professionnels. Dans une même perspective, on remarquera, exception faite des biens professionnels et du génie climatique, la quasi-absence de recours des entreprises les plus innovantes aux laboratoires publics, universitaires ou non, ce qui démontre l'exclusion d'une réalité pourtant incontournable de l'espace industriel, des structures de recherche universitaire, et cela est préoccupant. On peut dès lors, et en fonction des résultats, avancer deux types d'explications. En premier lieu, il est clair que la multiplication des partenaires pour ce qui concerne la R-D et les TPE les plus innovantes est 
révélatrice d'un besoin d'accroissement de la capacité technologique de la part de ces entreprises. Elles se retrouvent devant des seuils que seule une coopération avec d'autres structures leur permet de franchir. Il y a donc, comme nous l'avons déjà souligné, captation de ressources en fonction des enjeux mais cette captation révèle peut-être plus qu' une volonté «fédérative » ou « coopérative », un comportement opportuniste, les entreprises les plus innovantes étant celles qui s'avèrent les plus capables et les plus performantes dans cette stratégie de captation.

Caractère opportuniste qui donne à la structuration des réseaux un caractère éphémère, ceux-ci évoluant sans cesse en fonction des ressources disponibles. Benassi (1993) montre bien que ce qui importe lorsqu' on examine le problème des réseaux, ce n'est pas tant la densité des relations qu'y nouent les entreprises mais la possibilité qu'elles ont d'activer des ressources distribuées en fonction des problèmes et des occasions. Ainsi, le moindre recours à l'ANVAR de la part des entreprises les plus innovantes corroborerait cette hypothèse, les ressources captables de cet organisme étant, en dernier lieu et dans le cadre d'enjeux technologiques plus élevés, limitées ou évaluées comme telles.

Dans une même perspective, la non variation des scores de la catégorie relations avec les autres partenaires semblerait traduire une claire perception des entreprises, des ressources existantes, captives si l'on peut dire et de leur quasiintégration dans des trajectoires technologiques, quelles qu'elles soient. La plus ou moins grande intensité innovante des secteurs agit sur l'identification des centres de ressources et sur les stratégies de captation ultérieure. De la sorte, et en second lieu, l'opportunisme est à double détente. Dans un premier temps, il consiste à utiliser des centres «naturels » et de proximité géographique ou de métier et, dans un second temps, il va plutôt s'agir de repérer, au-delà des partenariats naturels, ce qui dans l'environnement peut apparaître comme une ressource mobilisable. C'est seulement à ce niveau que vont se distinguer les entreprises les plus innovantes, et ici la variable formation (Julien et Marchesnay, 1995) joue un rôle majeur. Contrairement à ce qu'avancent De Barry et Savoye (1993), les TPE innovantes ne se distinguent pas tant des moins innovantes par leur capacité à s'insérer dans des réseaux, mais plutôt par leur aptitude à saisir l'information nécessaire au bon moment, ce qui n'est pas la même chose. Cette capacité est en partie déterminée par le niveau de formation qui agit sur la faculté à rassembler les ressources, à les repérer mais aussi à être repérées. En effet, la nature des relations interorganisationnelles dépend des ressources internes possédées par les TPE, ressources qui proviennent en partie du secteur. Ces ressources vont induire des trajectoires d'innovation très différenciées et des stratégies interorganisationnelles, elles aussi, différenciées, par exemple de la simple sous-traitance de capacité au partenariat. De la même façon, on peut remarquer que les entreprises les plus innovantes du secteur le plus innovant (biens professionnels) se distinguent de celles moins innovantes non pas par un recours plus marqué aux « centres naturels » et institutionnels, mais 
par leur capacité à nouer des liens avec d'autres entreprises, notamment au regard de la sous-traitance de l'activité de R-D. Cette attitude s'explique en partie par la structure technologique particulière de ce secteur, mais elle peut s'expliquer aussi par le plus grand opportunisme des entreprises où l'on substitue à la notion de contrôle sur l'organisation la notion d'autonomie (dont le degré dépendra de la capacité de l'entreprise à capter des ressources utiles). Dans cette perspective, l'autonomie (et non plus l'indépendance) se trouve toujours liée au renforcement de la capacité technologique donc, en dernier lieu, plus à une capacité concurrentielle qu'à cette notion de contrôle sur l'organisation qui constitue un frein évident aux stratégies d'externalisation. C'est à ce niveau qu'il importe de ne pas confondre deux problèmes, soit le système de valeurs des dirigeants des TPE et leur relation à l'environnement. Les dirigeants des TPE les plus innovantes n'ont pas, quels que soient l'activité ou le degré d'innovation, un système de valeurs différent de celui des entreprises les moins innovantes. Ce qui les différencie, par contre, c'est précisément leur ouverture sur l'extérieur, qui influera sur la réceptivité de la TPE à l'innovation.

En ce sens, la relation avec l'extérieur est aussi bien une cause qu'une conséquence du comportement innovant. Là encore, comme le montrent les différents tests effectués, la variable «formation » est explicative de ce degré d'ouverture.

Le particularisme de la TPE doit donc être nuancé ; néanmoins, il est évident que certaines spécificités apparaissent et qu'il faut en tenir compte lorsque, par exemple, on envisage les politiques d'aide à l'innovation. De même, il faut relativiser certaines solutions par trop prescriptives et pas toujours empiriquement fondées, et nous pensons ici aux réseaux.

\section{Bibliographie}

AMENDOLA, M. et J.L. GAFFARD (1988), La dynamique économique de l'innovation, Paris, Economica.

ARCHIBUGI, D. (1988), "In search of a usefull measure of technological innovation », Technological Forecasting and Social Change, vol. 34, no 3, p. 253-277.

ARChIBUgi, D., S. CESARATTO et G. SirILli (1991), « Sources of innovative activities and industrial organization in Italy », Research Policy, vol. 20, p. 299-313.

ARCHIBUGI, D. et M. PIANTA (1994), "Innovation, patents and technological strategies », OECD Workshop, Paris, 8-9 décembre.

ARChibugi, D., R. Evangelista et R. Simonetti (1995), «Concentration, firm size and innovation : evidence from innovation costs », Technovation, vol. 2, $\mathrm{n}^{\circ} 1, \mathrm{p} .1-11$.

ARGYRIS, C. et D.A. SCHÖN (1978), Organizational Learning : A Theory of Action Perspective, Reading, Massachusetts, Addison-Wesley Publishing.

AUDRETSCH, D. et M. VIVARELli (1994), « Small firms and R-D spillovers : evidence from Italy ", Revue d'économie industrielle, $\mathrm{n}^{\circ}$ 67, $1^{\mathrm{er}}$ trimestre, p. 225-235. 
AXELSON, B. et G. EASTON (1992), Industrial Networks : A New View of Reality, Londres, Routledge.

BARDEN, P. et P. GOOD (1989), Information flows into industrial research, Londres, Gest.

BenASSI, M. (1993), Della Gerarchia Alla Rete, Milan, Etaslibri.

BOUVIER-PATRON, P. (1996), «Un concept de réseau pour analyser l'organisation industrielle», dans J.L. Ravix (dir.), Coopération entre les entreprises et organisation industrielle, Paris, Éditions CNRS, p. 171-190.

COMMISSARIAT GÉNÉRAL DU PLAN (1993), Recherche et innovation, le temps des réseaux, Paris, La Documentation française.

COMMISSARIAT GÉNÉRAL DU PLAN (1994 ), Intelligence économique et stratégie des entreprises, Paris, La Documentation française.

COHEN, W.M. et D.A. LEVINTHAL (1989), «Innovation and learning : the two faces of R-D », Economic Journal, vol. 99, p. 569-596.

CoOmbs, R., A. Richards, A. SAVIOTti et P.P. WALSh (dir.) (1996), Technological Collaboration, Cheltenham, E. Elgar, 232 p.

DE BANDT, J. (1996), «Coopération, accords interentreprises, concurrence », dans J.L. Ravix (dir.), Coopération entre les entreprises et organisation industrielle, Paris, Éditions CNRS, p. 195-230.

DE BARRY, C. et B. SAVOYE (1993), «Les petites entreprises innovantes », INSEE Première, $\mathrm{n}^{\mathrm{0}} 268$, juillet.

Dodgson, M. (1996) «Learning trust and interfirm technological linkages : some theoretical associations », dans R. Coombs, A. Richards, P.P. Saviotti et V. Walsh (dir.), Technological Collaboration, E. Elgar, p. 54-75.

Dodgson, M. et R. Rotwell (dir.) (1994), The Handbook of Industrial Innovation, Cheltenham, E. Elgar, 453 p.

Dussauge, P. et B. Ramanantsoa (1987), Technologie et stratégie d'entreprise, Paris, McGraw-Hill.

GAMBARDELLA, A. (1992), « Competitive advantage from in-house scientific research : the US pharmaceutical industry in the 1980s' », Research Policy, no 21, p. 391-407.

GREPME (1994), Les PME, Bilan et perspectives, Ouvrage collectif coordonné par P.-A. Julien, Paris, Economica Poche, p. 437.

Griliches, Z. (1984), R-D, Patents and Productivity, Chicago, University of Chicago Press.

GRILICHES, Z. (1990), «Patent statistics as economic indicators : a survey », Journal of Economic Literature, vol. 28, $\mathrm{n}^{\circ} 4$, p. 1661-1797.

GuELleC, D. (1993), Innovation et compétitivité, INSEE Méthodes, $\mathbf{n}^{\text {os }} 37-38$, Paris, Economica.

JOHANNINSON, B. (1993), «Entrepreneurs as learners, beyond education and training », dans H. Klandt et D. Muller-Boling (dir.), Internationalizing Entrepreneurship Education and Training, Koln-Dortmund, Förderkreis Gründungs-Forschung, p. 95-108. 
JULIEN, P.-A. et M. MARCHESNAY (1995), L'entrepreneuriat, Paris, Economica, p. 47-48.

KAMINSKI, P. (1995), «Comment aider la TPE de technologie », Cahier de recherche IRG, $\mathrm{n}^{\circ} 95.08$.

KIM, D.H. (1993), «The link between individual and organization learning », Sloan Management Review, p. 37-50.

KLEINKNECHT, A. et J.O.N. REIJNEN (1991), « More evidence on the undercounting of small firms RxD », Seo Overdruk, no 56.

KLINE, S. et N. RoSENBERG (1986), «An overview of innovation», dans R. Landau et N. Rosenberg (dir.), The Posititive Sum Strategy, Washington, National Academy Press, p. 275-305.

LE BAS, C. (1995), Économie de l'innovation, Paris, Economica.

LE BAS, C. et A. TORRÉ (1993), « Survey sur les surveys d'innovation, une première évaluation des enquêtes d'innovation européennes ", Revue d'économie industrielle, $\mathrm{n}^{\circ} 65$, $3^{\mathrm{e}}$ trimestre, p. 80-85.

LECOQ, B. (1989), Réseaux et système productif régional : contenu, portée et fondements théoriques du concept de réseau, CRER Dossier $n^{\circ} 23$, Université de Neuchâtel.

LE MEUN, G. (1993), «Les dirigeants de PME : autodidaxie ou autopraxéologie », Éducation permanente, $\mathrm{n}^{\circ} 114$, mars.

Marchesnay, M. (1992), «La PME : une gestion spécifique », Problèmes économiques, $n^{\circ} 2276,20$ mai.

MARCHESNAY, M. (1994), « Le management stratégique », Cahier du GREPME, p. 133-162.

MÉDUS, J.L. et J.C. PACITTO (1994), «L'innovation technologique dans la très petite entreprise : un premier bilan », Cahier de recherche IRG, $\mathrm{n}^{\circ} 94.11$.

MILLER, D. et P.H. FRIESEN ( 1982), «Innovation in conservative and entrepreneurial firms : two models of strategic momentum », Strategic Management Journal, vol. $3, \mathrm{n}^{\circ} 1$, p. 10-25.

MinguZZI, A. et R. PASSARO (1995), «L'apprentissage comme facteur d'innovation des entreprises ; enquête et exploration dans les petites entreprises italiennes », Actes du Deuxième Congrès international francophone Innovation et organisation des $P M E$, Paris.

PACHÉ, G. et C. PARAPONARIS (1993), L'entreprise en réseau, Paris, Presses universitaires de France.

PACITTO, J.C. (1996), «La très petite entreprise innovante : entre réalités et fictions », Cahier de recherche IRG, $\mathrm{n}^{\circ}$ 96.12.

PECQUeUR, B. (1989), Le développement local, Paris, Syros Alternatives.

PERrIEN, C. (1994), «Logique de perfectionnement des dirigeants de PME-PMI », Revue ANDCP-Personnel, $\mathrm{n}^{\circ} 351$, juin, p. 73.

Polanyi, M. (1966), The Tacit Dimension, Londres, Routledge and Kegan Paul.

Quelin, B. (1996), «Coopération inter-entreprises et Création de Ressources », dans J.L. Ravix, (dir), Coopération entre les entreprises et organisation industrielle, Paris, Éditions CNRS, p. 111-140. 
RAVIX, J.L. (1996), Coopération entre les entreprises et organisation industrielle, Paris, Éditions CNRS.

RIZZONI, A. (1988), «Innovazione Tecnologica e Piccola Impresa : un Approcio Tipologico » Piccola Impresa, $\mathrm{n}^{\circ}$ 1, p. 67-89.

RIZZONI, A. (1994), «Technology and organization in small firms : an interpretative framework », Revue d'économie industrielle, $\mathrm{n}^{\circ}$ 67, p. 135-155.

Rosenberg, N. (1982), Inside the Black Box: Technology and Economics, Cambridge, Cambridge University Press.

ROTHWELL, R. (1994), «Industrial innovation : success, strategy, trends », dans M. Dodgson et R. Rotwell (dir), The Handbook of Industrial Innovation, Cheltenham, E. Elgar, p. 33-44.

SANTARELli, A. et A. STERLACCHINI (1990), «Innovation, formal vs informal R-D and firm size : some evidence from Italian manufacturing firms », Small Business Economics, vol. 2 , p. $223-228$

SCHERER, F.M. (1982), « Inter-industry technology flows and productivity growth », Review of Economics and Statistics, vol. 64, p. 627-634.

SCHERER, F.M. (1991), «Changing perspectives on the firm size problem », dans Z. Acs et D.B. Audretsch (dir.), Innovation and technological change : an international comparison, Ann Arbor, University of Michigan Press, p. 24-38.

SENKER, J. et W. FAULKNER (1996), « Networks, tacit knowledge and innovation », dans R. Coombs, A. Richards, P.P. Saviotti et V. Walsh (dir.), Technological Collaboration, E. Elgar, p. 76-97.

SERVICE DES STATISTIQUES INDUSTRIELLES (1994), L'innovation technologique dans l'industrie, Paris, Ministère de l'Industrie.

SMITH, K. (1989), « Towards a new system of innovation indicators », Paper for Innovation Indicators Conference, Oslo, Norvège.

STEWARD, F. et S. CONWAY (1996), «Informal networks in the origination of successful innovations », dans R. Coombs, A. Richards, P.P. Saviotti et V. Walsh (dir.), Technological Collaboration, Cheltenham, E. Elgar, p. 201-221.

TEECE, D.J. (1988), «Technological change and the nature of the firm », dans G. Dosi $e t$ al. (dir.), Technical Change and Economic Theory, Londres, Pinters Publishers.

ZaGOURAS, N. et K. PAPANAYOTOU (1995), «La petite unité innovante en Grèce », Actes du Deuxième Congrès international francophone de la PME, Paris, p. 175-189.

Williamson, O.E. (1985), Markets and Hierarchies : Analysis and Antitrust Implications, New York, Free Press. 